Part of Journal of Research of the National Bureau of Standards, Volume 27, September 1941

\title{
TESTING AND PERFORMANCE OF VOLT BOXES
}

\author{
By Francis B. Silsbee and Francis J. Gross
}

\section{ABSTRACT}

The apparatus and method used in the precise measurement of the ratio of volt boxes at the National Bureau of Standards are described in detail. The method is applicable with rated voltage applied to the volt box and has an accuracy approaching one part in 100,000. The results of such measurements on a number of volt boxes under various conditions of humidity and temperature are given as a guide to their use and an index of their accuracy.

Introduction

II. Method

III. The standard volt box _...

IV. The Lindeck potentiometer $\ldots \ldots \ldots$

V. Auxiliary features._.

VI. Test procedure

VII. Typical performance characteristics of volt boxes _ _ _ _ _ _ _ _ 278

1. Accuracy of manufacturer's adjustment of ratio

2. Secular change_._.

3. Seasonal variation of ratio

4. Effect of surface resistance of insulating panel on the ratio of

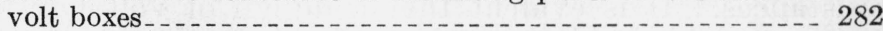

5. Effect of self-heating

VIII. Appendix I. Alternative test method _.

IX. Appendix II. Derivation of test schedule for calibrating the standard volt box . .

\section{INTRODUCTION}

A volt box, more appropriately called a "resistance potential divider," is a device commonly used to extend the range of a potentiometer by a definite factor so as to permit of the precise measurement of direct voltages which exceed the limited range of the potentiometer. Any error in this factor will of course enter directly into the measurement of voltage, and in cases where the volt box is used in connection with the testing of wattmeters or watthourmeters, into the measurement of the commercially valuable quantities, electric power and energy.

A volt box consists of a number of resistors connected in series and provided with binding posts so that the unknown voltage to be 
measured can be connected to the whole of the series circuit (or to some relatively large fraction thereof), while the potentiometer is connected to measure the potential drop between the terminals of a smaller part of the circuit thus energized. In the absence of electric leakage, the ratio of the unknown voltage to the potential drop measured by the potentiometer is evidently equal to the ratio of the resistance of the whole circuit to that of the portion connected to the potentiometer. An accurate determination of this ratio thus constitutes a test of the volt box.

It will be seen that such a test involves only the ratio of two resistances and not their absolute values. Consequently a test of a volt box does not necessarily involve any measurements of resistance in terms of the International Ohm, but only the much easier process of relative measurement. A further result of this fact is that certain types of variation which affect all parts of the circuit equally, such as changes in room temperature, tend to cancel their effects leaving only very small residual errors. Theoretically it would be desirable if all parts of the circuit were formed of wire of the same size, composition, and treatment. Practical difficulties in making adjustments may arise if the length of fine wire required for any of the low ranges becomes too short. Sometimes this difficulty can be met by using a short length of larger manganin wire at one end of the small fine-wire coil and making the final adjustment on this larger wire. Also, the construction of coils of very different resistance often produces differences in cooling surfaces and thus in load coefficient.

Volt boxes are, therefore, subject to a number of sources of error, two of which depend upon the applied voltage. First, the heat developed in the resistors when subjected to the operating voltage (sometimes as much as 1,500 volts) may create temperature differences and hence vary the relative resistance of the component parts. Second, any leakage over the insulating supports of the individual resistors will make the current in some of them differ from that in others, so that the ratio of voltages will not be the same as the ratio of resistances. It is evident that a design of volt box in which selfheating trouble has been made negligible by the use of a circuit of very high resistance will be particularly liable to leakage trouble, and vice versa. In view of the facts that in general the resistance of leakage paths does not obey Ohm's law, and that the resistance of manganin does not vary linearly with temperature, it is obviously very desirable that a volt box be tested under conditions as nearly as possible the same as those under which it is used, and in particular, that it be tested at operating voltages.

It is the purpose of this paper to describe the equipment used at the National Bureau of Standards for making such tests, and also to give typical data showing the performance of present eommercial volt boxes, as a guide to other laboratories which may be called upon for similar testing work.

\section{METHOD}

The method of test here used is based on a comparison of the ratio of the volt box under test with that of a standard volt box of the same nominal range, the ratio of which has been determined by methods to be described. Of the various possible methods for making the intercomparison, the following has been found most flexible and convenient. 
The two volt boxes are connected in parallel between junction points $A$ and $B$, as shown in figure 1 , and supplied with rated voltage, $V$. The corresponding terminals, such as $S_{3}$ and $X_{3}$ or $S_{2}$ and $X_{2}$, are then at very nearly equal potentials. If the small potential differences between these terminals are measured and denoted by $v_{1}, v_{2}, v_{3}$, and $v_{4}$, respectively, then the ratio, $F_{X}$, of the unknown is given by the equation

$$
F_{X}=F_{S}\left(1+\left[\left(v_{4}-v_{1}\right) / V\right]-F_{S}\left[\left(v_{3}-v_{2}\right) / \mathrm{V}\right]+\ldots\right),
$$

in which terms of the second and higher order in $F_{S}\left(v_{3}-v_{2}\right) / V$ and $\left(v_{4}-v_{1}\right) V$ have been neglected. Here any $v$ is to be taken as positive if the $X$ terminal is positive with respect to the $S$ terminal when $B$

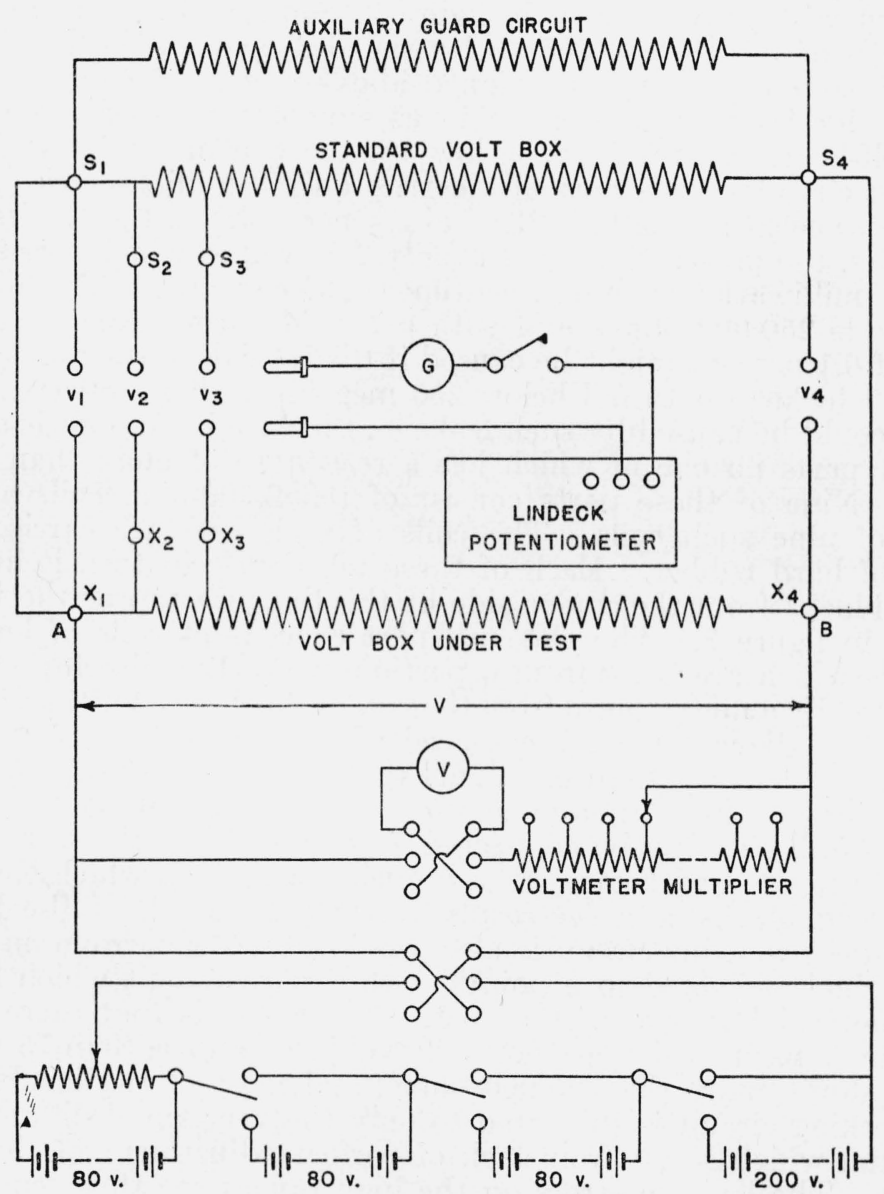

FigURE 1.-Diagram of circuit used to intercompare volt boxes by the first method.

is positive with respect to $A$. The quantities $v_{4}$ and $v_{1}$ arise only from the resistance of leads and contacts and are always small (seldom more than 250 microvolts at rated voltage); $v_{2}$ is also usually small, and differs from $v_{1}$ only as the result of the resistance of connectors within the volt boxes. Furthermore $v_{3}$ is small (seldom exceeding 
1,000 microvolts) unless the volt box under test is seriously out of adjustment. Consequently in measuring the small potential differences, $v$, and the applied voltage, $V$, only a moderate relative accuracy is sufficient to give a high precision in $F_{X}$. The applied voltage, $V$, is conveniently measured with a voltmeter, but the small potential differences, $v$, should be measured with a low-range potentiometer.

The three sections which follow describe in some detail the main components of the equipment used in this method at the National Bureau of Standards.

\section{THE STANDARD VOLT BOX}

The apparatus used at the National Bureau of Standards as a standard volt box is shown in figure 2, and its ranges are listed in column 8 of table 1 (p. 287). It was designed so as to minimize the two sources of error mentioned above and at the same time to provide for its own calibration in as simple a manner as possible. The self-heating error is kept low by the use of a high-resistance working circuit. This normally carries only 3 milliamperes (that is, its resistance corresponds to $333 \frac{1}{3} \mathrm{ohms}$ per volt) so that at its rated 750 volts, the power dissipated is only 2.25 watts, which corresponds to 0.25 milliwatt per square centimeter of coil area. The total resistance is $250,000 \mathrm{ohms}$, and with a box of normal construction an error of 0.1 percent might be caused if the insulation resistance of the effective leakage path fell below 250 megohms. To reduce the error which could be caused by such leakage, the working circuit is divided into 11 parts no one of which has a resistance of more than 25,000 ohms. Nine of these parts consist of ten 2,500-ohm coils and the tenth of nine such coils. The coils of each part are carried by a panel of hard rubber. Each of these ten panels in turn is hung by metal blocks from the under side of the Bakelite top of the box, as shown in figure 3 . The eleventh part consists of coils of lower resistance which are hung from a portion of the Bakelite top which is separated by a metal guard from the rest of the box. The hard-rubber panels for adjacent parts are spaced with a clearance of $5 \mathrm{~mm}$, and any surface leakage from one panel to the next is intercepted by the supporting blocks. An auxiliary or "guard" circuit, corresponding sections of which can be connected in parallel with the working circuit, is provided with taps at appropriate points to which the metal supporting blocks are electrically connected. Each of the binding posts of the working circuit is also provided with a surrounding metal guard ring connected to a point on the guard circuit which has the same potential as that of the post. The result is that there can be no leakage paths which are normally subject to more than 75 volts or which shunt more than 25,000 ohms, and most of the insulation of the working circuit is subjected to only the very small differences of potential which result from lack of perfect adjustment of the guard circuit. The leakage errors on the high ranges are thus reduced by a factor of more than 100 . The guard circuit has a nominal resistance of $266 \% 3$ ohms per volt.

The calibration of the standard volt box requires the determination of the resistances of various parts of the working circuit relative to one another. The process consists in, first, the intercomparison of a group of sections of nominally equal resistance and then the connection 


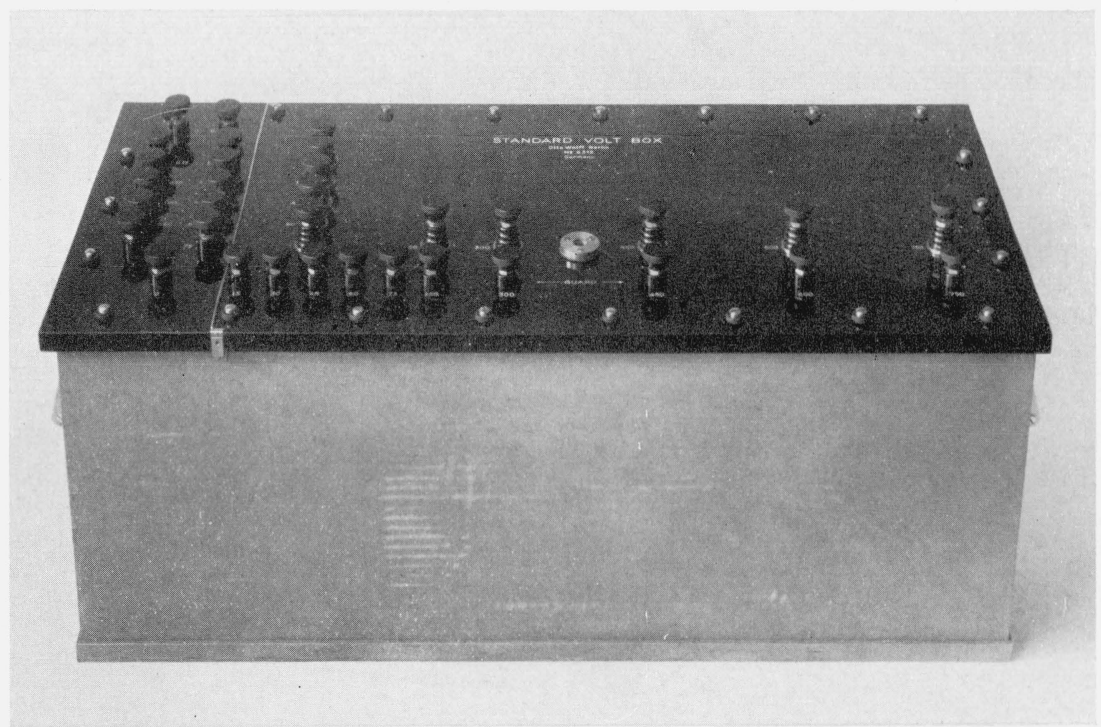

Figure 2.-The standard volt box. 
of these equal sections in series to form the first section of a group of larger denomination. If the resistance of the first section of a group of five is arbitrarily taken as a base, the first four intercomparisons give the separate resistances of the next four sections relative to this base. The addition of these values gives the resistance relative to the base of the entire group connected in series. This group itself constitutes the first section of the next group, the resistances of which are thus determined in terms of the original base. To minimize the number of individual sections while retaining the series of nominal ratios used in most commercial volt boxes, it was found desirable to use

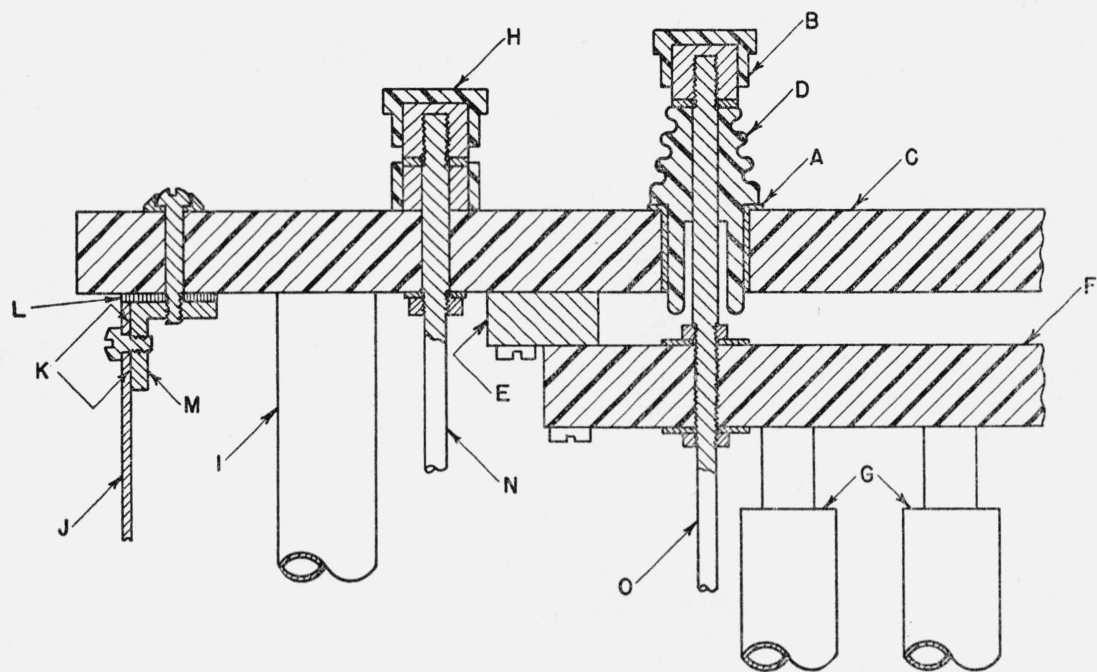

Figure 3.-Cross section through a portion of the standard volt box.

$A$, Metal guard ring connected to guard circuit.

$B$, A terminal of the standard volt box.

$C$, Bakelite top panel.

$D$, Insulator bushing.

$E$, Brass support for panel $F$. $E$ is connected to the guard circuit.

$F$, Hard rubber panel.

$G$, Two resistance coils of the working circuit.

$H$, A guard-circuit terminal.
I, A resistance coil of the guard circuit.

$\vec{J}$, Air-tight metal box.

$K$, Sweated air-tight joint.

$L$, Rubber gasket.

$M$, Brass rod, which extends to bottom of $I$. A tap lead of guard circuit connects to bottom of $N$.

$O$, Brass rod extending to bottom of $G$. A tap from the working circuit connects to bottom of $O$.

groups containing alternately five and two nominally equal sections. Thus the first group consists of five sections of $50 \mathrm{ohms}$ each which are rated at 150 millivolts each. These sections connected in series form the first $250-0 h m$ section of the second group of two sections. These two $250-\mathrm{ohm}$ sections in series form in turn the first 500-ohm section of the third group, which again consists of five sections. This scheme is carried along to the highest sections of $50,000 \mathrm{ohms}$ each, which are rated for a potential drop of 150 volts.

The most convenient way of intercomparing the resistance of the sections of any one group is to substitute one after another in the unknown arm of a Wheatstone bridge as shown in figure $4(A$ and $B)$. This bridge circuit must be definite and sensitive to the full percentage accuracy desired in the volt-box calibration, but the corrections to its arms and the lead resistances ordinarily need not be known, provided they remain the same during the intercomparison of the sections of each group. 
The intercomparison of the lower voltage sections by this substitution method can readily be made with full rated voltage applied to the section under test. To apply full voltage $(150$ volts $)$ to the higher

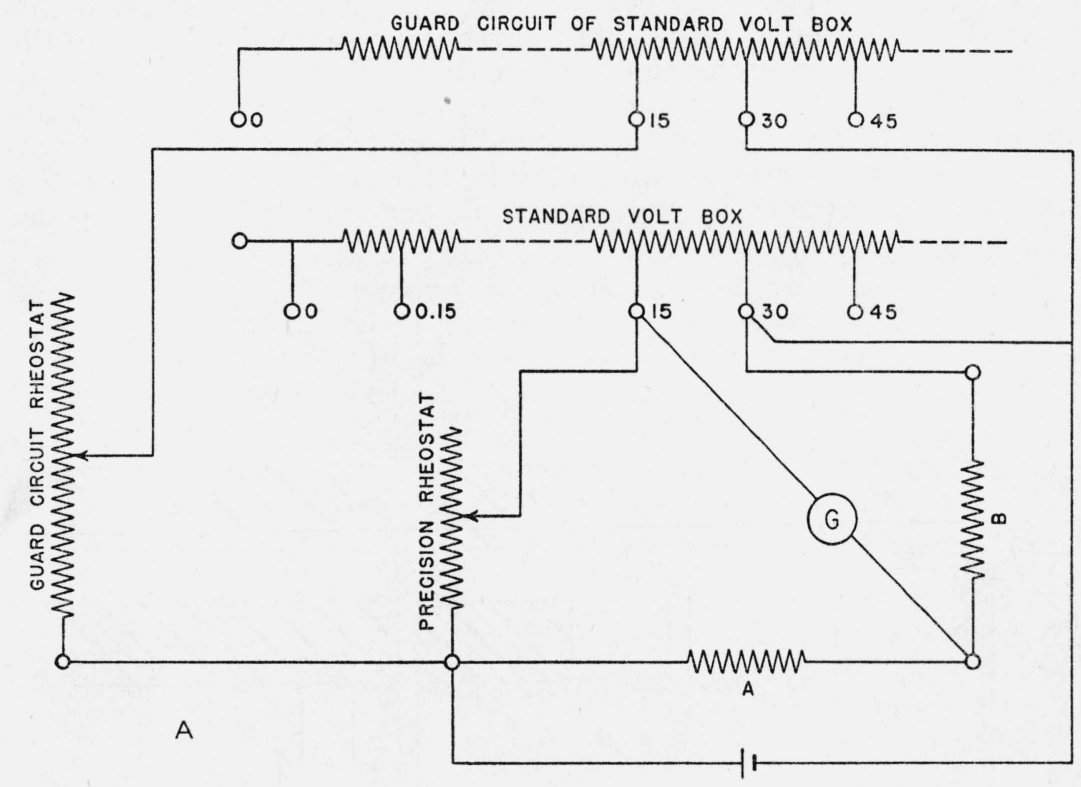

STANDARD VOLT BOX

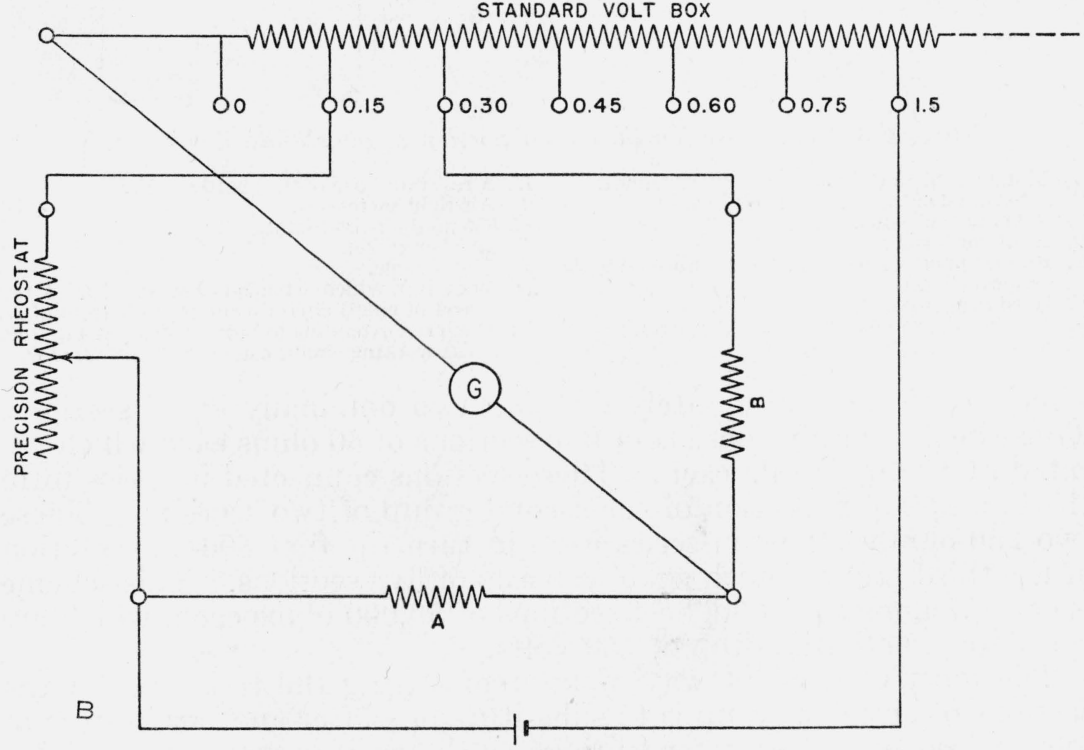

FIgURE 4.-Bridge circuits for calibrating standard volt box.
A, Higher sections.
$B$, Lower sections.

voltage sections with the bridge arrangement of figure $4(A)$ would require the use of a bridge arm of about $10,000 \mathrm{ohms}$, the resistance of which remains steady when dissipating 2.25 watts. Experiments 
using such an improvised arm have shown that the ratio of the 50,000ohm sections to the $500-\mathrm{ohm}$ section is the same at 300 volts and at 150 volts as at 30 volts, within 3 parts per million. Routine calibrations are therefore made at only 30 volts. In the case of the high-voltage sections, the measurement should be made with the corresponding section of the guard circuit energized by connecting it to the battery which supplies the bridge current, as shown in figure $4(A)$.

Table 1 (see p. 287) is the schedule used for computing the final volt-box corrections from the bridge readings. In the table each value of $p$ is the observed resistance of the precision rheostat in the bridge circuit of figure $4(A$ and $B)$. The computed values of $s$ in column 7 are the corrections in parts per million which, when applied to the nominal ratio of the resistance of the range in column 8 to the resistance of the 0 to 0.15 range, will give the actual ratio. To obtain the actual ratio for any range in column 8 to any other range listed in that column, subtract the value of $s$ for the second range from that of the first and apply this difference as a correction in parts per million to the nominal ratio of the two ranges. The mathematical derivation of the equations of table 1 is given in appendix II.

The actual carrying out of a test by this procedure is very rapid, a complete test and computation for all ranges being obtainable in less than 3 hours. The sole theoretical error in the procedure as described arises from the resistance of the rods which lead from the junction points of the sections to the binding posts. The resistance of each rod is only $0.0006 \mathrm{ohm}$. If the rods are equal in resistance, the error will be equal to the resistance of two rods divided by the total resistance of the volt-box range used. Differences in rod resistance will cause errors equal to these differences divided by the resistance of the section measured. To eliminate this latter error in the measurements of the 50-ohm sections, the galvanometer and battery connections of the bridge circuit are made through the sections next adjacent to the one under test, as shown in figure $4(B)$, and thus the rods are put into arms of higher resistance. The effect of the rod resistance on the voltbox ratio is less than 3 parts per million.

As a measure of the precision readily attainable in this process of calibration, data obtained over a period of 12 years were examined and 27 successive independent determinations of the ratio of two sections of identical construction, namely that between the 450 - and 600-volt taps and that between the 600- and 750-volt taps, were tabulated. The standard deviation of these values from their mean was found to be 7 parts per million, the greatest individual deviation being 19 parts per million. This deviation, of course, includes any drift in the relative values of the coils involved as well as the errors in the setting up of their ratio.

The individual coils are wound with a single layer of silk-covered manganin wire, all coils being wound with wire from the same spool so as to minimize differences in the temperature coefficient of resistance of the various parts. Nevertheless, early data showed a tendency to a cyclical annual fluctuation in the ratio of the 750 -volt to the 1.5 -volt section amounting to about 30 parts per million. This is apparently the result of changing atmospheric humidity. To minimize such effects, a small air pump is arranged to maintain the average pressure within the standard volt box at $15 \mathrm{~mm}$ of water above atmospheric. Air from the pump passes through a drying vessel containing $\mathrm{CaCl}_{2}$ 
and is thus thoroughly dried before entering the box. A rubber gasket between the metal box and the Bakelite top seals the joint sufficiently so that an air flow of about $0.1 \mathrm{~m}^{3}$ per 24 hours is enough to maintain the pressure differential. Since the installation of this dry-air supply, the cyclic fluctuation has been very small.

\section{THE LINDECK POTENTIOMETER}

The potentiometer generally used for measuring the small voltages, $v_{1}, v_{2}, v_{3}$, and $v_{4}$, referred to in section II is of the Lindeck type. ${ }^{1}$ Figure 5 is the circuit diagram of this instrument. In this device a current

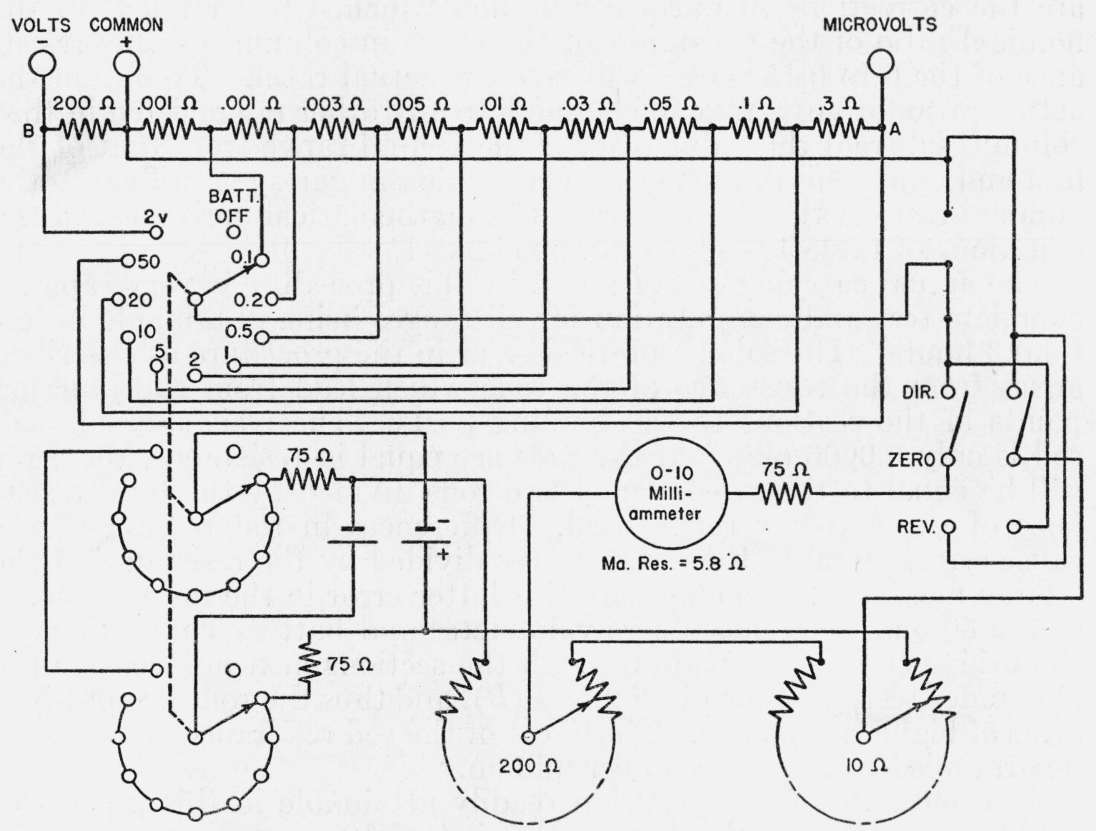

FIGURE 5.-Circuit of the Lindeck potentiometer.

When intercomparing volt boxes, the terminals labeled "common" and "microvolts" are used. When interchecking the calibrations of the potentiometer and the voltmeter, the terminals labelled "common" and "volts" are used.

measured by the milliammeter passes through a selected portion of the standard resistor, $A B$, and this current is adjusted until the voltage drop in the standard resistor is exactly equal and opposite to the voltage to be measured, as indicated by the external galvanometer (shown at $G$ in fig. 1), which is used as a null indicator.

The milliammeter has a scale length of approximately $15 \mathrm{~cm}$ and is equipped with a parallax mirror. It requires $10 \mathrm{ma}$ for full-scale deflection.

The standard resistance sections between $A-B$ in figure 5 are made of manganin and are so constructed that the temperature rise is negligible even with the current of 8 amperes which is used to calibrate them on a Kelvin double bridge. Their resistance should be adjusted

1 J. C. Poggendorff, Ann. Phys. Chem. 54, 161 (1841); Lindeck and Rothe, Z. Instrumentenk. 20, 293
(1900); H. B. Brooks and A. W. Spinks, BS J. Research 9, 781 (1932) RP506. This last paper contains a brief summary of the two references above and a discussion of basic potentiometer circuits. 
to a precision somewhat better than that to which the milliammeter can be read.

Since the resistance of the galvanometer circuit may vary from a low value for measurements of $v_{1}, v_{2}$, and $v_{4}$ up to several hundred ohms in the measurement of $v_{3}$, the time required to obtain a reading can be shortened by providing manganin rheostats in series and in parallel with the galvanometer, so that the instrument can be critically damped in all four measurements. In operation, the voltage applied to the volt boxes, and the potentiometer current are reversed simultaneously, so that the effect of any constant thermal emf in the galvanometer circuit or elsewhere is eliminated. By moderate care in using only copper and materials, such as manganin, which have a low thermal emf against copper, the residual emf is so small that fluctuations in it cause only negligible errors.

The external galvanometer has a sensitivity of $3 \mathrm{~mm}$ per microvolt at $1 \mathrm{~m}$ distance, with a critical external damping resistance of $200 \mathrm{ohms}$ and a period of 5.8 seconds.

\section{AUXILIARY FEATURES}

All of the wires in the measuring circuits are run in shielded cable, and each shield is maintained at the same potential as the wire inside it by means of the auxiliary "guard" circuit of the standard volt box mentioned in section III above. The galvanometer and the Lindeck potentiometer are mounted on metal guard plates which are so located as to intercept any possible leakage currents. When voltage $v_{1}, v_{2}$, or $v_{3}$ is being measured, these guard plates are connected to the low-voltage terminal of the auxiliary guard circuit of the standard volt box; but when $v_{4}$ is measured, these plates are connected to the high-voltage terminal of the guard circuit. A special plug-connector panel is used to connect the Lindeck potentiometer circuit to measure $v_{1}, v_{2}, v_{3}$, and $v_{4}$, and this panel is designed so that guard plates intercept leakage currents between the different measuring circuits.

For test voltages from 400 to 750 volts the batteries shown in figure 1 are replaced by a motor-driven d-c generator. The driving motor and the field of the generator are energized by means of batteries.

With this equipment, 750 volts is the nominal limit at which volt boxes are tested. However, experiment has shown that the calibration of the standard volt-box can be relied upon up to 1,500 volts, and by special arrangements the generator can be connected in series with a group of batteries to obtain this voltage.

Since this method of calibrating a volt box involves the ratio of the small voltages measured by the potentiometer to the larger voltage measured by the voltmeter, it is necessary that the readings of these two instruments be mutually consistent, though neither need be accurate. Means were therefore provided for readily intercomparing them. This is done by applying the same voltage to the voltmeter and the standard volt box and measuring the voltage drop across the 0 to 1.5-volt range of the standard volt box with the Lindeck potentiometer. The "volts" and "common" terminals (see fig. 5) of the potentiometer are used. Knowing the volt-box ratio, the agreement of the two instruments can be readily checked. Since the volt- 
meter is provided with a shunt circuit around the moving element to provide temperature compensation in the manner described by Brooks, ${ }^{2}$ it was found convenient to incorporate in this shunt circuit a small rheostat which can be used to adjust the current through the moving element, so that the calibration of the voltmeter may be made consistent with that of the potentiometer in spite of any small changes in either instrument.

\section{TEST PROCEDURE}

When a volt box is received for test at the National Bureau of Standards, the resistances between its terminals are first measured with a Wheatstone bridge, in order to detect any defective resistance coils before full voltage is applied to them. Data from these measurements can also be used to compute the approximate ratio. The volt box is then connected into the circuit of figure 1 , and voltage is applied. Voltages $v_{1}, v_{2}, v_{3}$, and $v_{4}$ are then measured successively, by adjusting the Lindeck potentiometer until the simultaneous reversal of polarity of the applied voltage, $V$, and of the potentiometer does not change the galvanometer deflection. This latter procedure makes negligible those errors that might be caused by any constant thermal emf in the galvanometer circuit. Because of the self-heating effect, to be described in section VII-5, the volt box is then allowed to warm up until the measured value of $v_{3}$ becomes reasonably constant. If the volt box has more than one range, the highest range is measured first and then the other ranges are measured in order of decreasing ratio. This procedure requires only one warming-up period for the entire box.

A very effective check on the accuracy of the measurements is obtained by making two independent measurements of each ratio of the volt box under test, using two different standard volt-box connections which give the same ratio. Examples of standard volt-box terminal arrangements which give the same ratio are: $0-1.5-150,0-3-300$, 0-6-600, and 0-7.5-750.

Ratios of the tested volt box are computed by means of the equation given in section II. If for any range the total change in ratio caused by self-heating is greater than the precision ( 0.01 percent) to which results are reported, then the observed change and the time required to complete it are reported.

\section{TYPICAL PERFORMANCE CHARACTERISTICS OF VOLT BOXES}

Although the technical literature abounds with articles on potentiometers and much has been done to improve their accuracy, volt boxes are mentioned only incidentally and with the exception of manufacturer's guarantees, no literature appears to have been published which discusses the factors affecting the accuracy of the voltage ratio of volt boxes. Perhaps the reason for this lack of published information is that up to the present time the accuracy of the measurements in which volt boxes have been used has not been noticeably limited by any variation in the voltage ratio of the latter. In view of this situation, a somewhat detailed discussion is given below of the

\footnotetext{
${ }^{2}$ H. B. Brooks, J. Research NBS 17, 523 (1936) RP926.
} 
factors which affect the voltage ratio so that those who use volt boxes may know what factors may affect measurements of a given precision.

At the time of the construction of a volt box the departure of its actual voltage ratio from the nominal value will depend upon the care with which the manufacturer adjusts the resistances of the various coils. Thereafter the departure of the voltage ratio from the intial value may depend upon (a) secular change of the resistance of the coils, (b) ambient temperature, (c) temperature changes caused by self-heating, (d) slow seasonal variations in ratio caused by seasonal changes in relative humidity, and (e) changes in insulation resistance between terminals and between the coil studs.

\section{ACCURACY OF MANUFACTURER'S ADJUSTMENT OF RATIO}

Figure 6 indicates the accuracy oi the manufacturer's adjustment of voltage ratio on 86 volt boxes teited at the National Bureau of

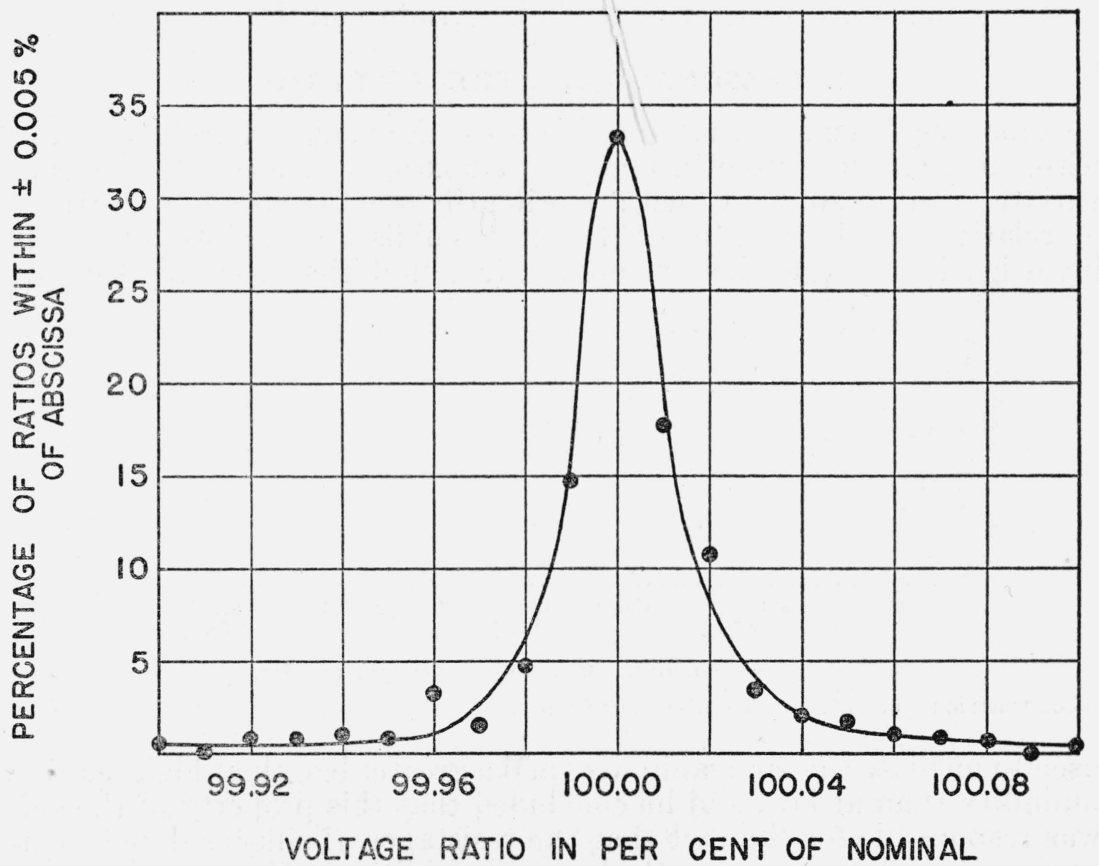

FIGURE 6.-Accuracy of the manufacturer's adjustment of voltage ratio.

This curve represents data on 86 volt boxes tested at the National Bureau of Standards between 1927 and 1940. In computing the curve each range of a multiple-range volt box was considered separately, so that there were 413 ranges tested.

Standards between the years 1927 and 1940. For each plotted point in this figure the ordinate represents the percentage of volt-box ranges tested the ratio of which was within \pm 0.005 percent of the ratio indicated by the abscissa. If the volt box was tested on more than one occasion, only the results of the initial test were used in locating this curve. Curves of this type for individual ranges had the same

$404687-41-5$ 
general shape and distribution of points as the composite curve shown here. All of the volt boxes represented in this curve were for 1.5-volt potentiometers. However, a curve of this type based on tests of a small number of volt boxes used with 150 -millivolt deflection potentiometers displayed a similar shape and distribution of points.

\section{SECULAR CHANGE}

One would expect that the secular change of ratio would be less than the secular change of resistance of the individual coils, provided that all of the coils are of similar construction. Data are available on seven volt boxes which have been sent in to the National Bureau of Standards for annual test over periods of from 6 to 10 years. Of these seven volt boxes, the voltage ratio of the 150 -volt to the 1.5 -volt range has varied 0.01 percent, or less, in six of them, and the ratio of the seventh has increased uniformly by a total of 0.04 percent in a 10-year period. The other ranges of each volt box in this group varied in the same manner as the 150-volt to the 1.5-volt range. It may be supposed that this performance is typical of high-grade volt boxes.

\section{SEASONAL VARIATION OF RATIO}

It has long been known that resistance coils of silk-covered manganin wire, such as those used in many volt boxes, are subject to seasonal variations in resistance which are primarily due to seasonal variations in relative humidity. In 1936 Dike $^{3}$ published the results of his investigation of the phenomenon. He found that the silk threads

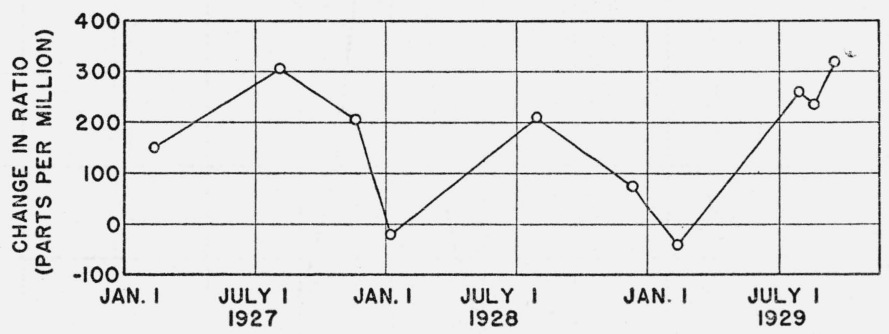

Frgure 7.-Seasonal variation of ratio for volt box $A$.

Construction features of $A$ are described under figure 8 .

used to insulate the manganin wire had a greater length at high relative humidity than at low, and he concluded that this property of the silk was responsible for the fact that the resistance of silk-insulated manganin wire coils is greater at the higher humidities. He also found that if such coils were coated with certain waxes and suddenly changed from an atmosphere of one relative humidity to that of another, the resistance change would require approximately 3 weeks to complete, but that coils not coated with wax required less than 24 hours although the magnitude of the change in resistance was the same. The percentage change of resistance is greater the finer the wire.

Figure 7 shows the seasonal variation in ratio for a volt box in which the wire size is much larger on the low-voltage coil than on the coils nearer the high-voltage end. Dike's theory explains this curve very well, because from that theory we would expect the humid summer

' P. H. Dike, Rev. Sci. Instr. 7, 278 (1936). 
air to produce a greater percentage increase in the resistance of the fine wire coils than in the coarser wire of the low-voltage coil and hence make the voltage ratio higher during the summer months than in the drier winter months. It should be noted that artificial heating in winter causes the relative humidity indoors to be materially lower than that of the outside air. This curve should not be interpreted

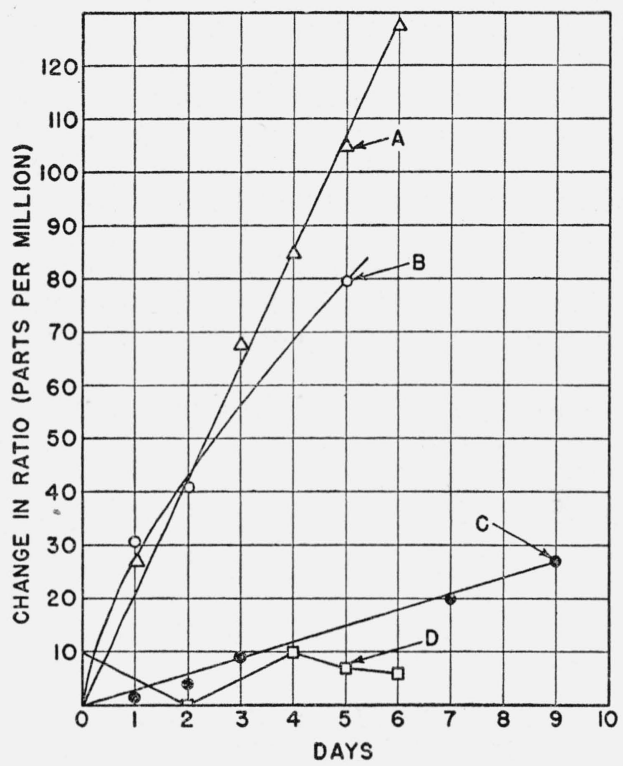

FIGURE 8.-Drift of voltage ratio after a sudden increase in relative humidity.

Each volt box stood for several weeks in a room having a relative humidity between 25 and 40 percent, and was then placed in a closed cabinet in which a saturated solution of sodium chloride maintained a relative humidity of 75 percent. Each curve represents measurements made on the 150-volt to the 1.5-volt range of a volt box, with the exception of the curve for volt box $A$ on which the 150 -volt to the 0.15 -volt range was measured.

Volt box $A$ has a resistance of 40 ohms per volt, its wood spools are wound with silk-insulated wire, and the diameter of the wire on the low-voltage coils is approximately 10 times that of the wire on the highvoltage coils.

Volt box $B$ has a resistance of $200 \mathrm{ohms}$ per volt, its metal spools are wound with silk-covered wire, and the ratio of the diameter of the wire in the low-voltage coil to that of the wire in the high-voltage coil is intermediate between that of volt boxes $A$ and $C$.

Volt box $C$ has a resistance of 2623 ohms per volt, its wood spools are wound with silk-covered wire, and the diameter of the wire in the low-voltage coil is twice that of the wire in the high-voltage coils.

Volt box $D$ has a resistance of 333 ohms per volt; its metal spools are wound with enameled wire which is insulated with a patented mixture of silk and cotton, and the ratio of the diameter of the wire in the low. voltage coils to that of the wire in the high-voltage coils is approximately the same as that of $C$.

The coils of all four volt boxes are wax-coated.

as a typical curve for all volt boxes, since it probably represents an extreme type of construction among volt boxes built by reputable manufacturers.

To obtain additional data on the effects of humidity, an air-tight cabinet was built in which a volt box could be placed. A tray of saturated sodium chloride solution kept the relative humidity constant at 75 percent. Shielded leads were brought out so that the ratio of the volt box could be measured without opening the cabinet. Data for each of the curves in figure 8 were obtained by placing in this cabinet a volt box which for several weeks previously had been in an atmosphere having a relative humidity of less than 40 percent, and then periodically measuring the voltage ratio without removing 
the volt box from the cabinet. These ratio measurements were made on the 150 -volt range of each volt box with only 30 volts applied so as to make any self-heating effects negligible.

Volt boxes $A, B$, and $C$ have coils wound with silk-covered manganin wire. The manganin wire of volt box $D$, however, is insulated with a patented ${ }^{4}$ combination of silk and cotton, which is claimed to cause much less seasonal variation of resistance with humidity. The improvement is obvious from the curves.

These curves for volt boxes in which silk-covered manganin is used bear out two implications from Dike's paper. First, for a given voltage ratio the effect of a change in humidity will be least for the volt box having the fewest ohms per "volt, since such a box will usually have thicker wire; this would explain the small effect on the ratio of $C$ in figure 8. Second, the greater the difference between the wire diameters of the low-voltage and high-voltage coils, the greater will be the effect of humidity. This is shown by the curves for $A$ and $B$, where $A$ has one-fifth of the ohms per volt of $B$ but the greater difference in wire size.

After the data for figure 8 were obtained, volt box $A$ was kept in the cabinet for 4 weeks, and its ratio was found to have increased 0.048 percent in that time. Upon removing it from the cabinet to the dry air of the room, the ratio decreased exponentially, with a change of 0.029 percent the first week. Although the experiments reported above were made on a limited number of volt boxes, the results probably indicate the magnitude and character of the effects that would be experienced in the actual use of volt boxes.

\section{EFFECT OF SURFACE RESISTANCE OF INSULATING PANEL ON THE RATIO OF VOLT BOXES}

Some of the coil studs and binding post terminals of volt box $C$, which had the customary hard-rubber top, were disconnected from the coils and the insulation resistance between them was measured. Computations based on these measurements showed that surface leakage between coil studs and between terminals of a volt box would cause variations in the ratio of less than 5 parts per million for a relative humidity of 75 percent or less, provided the resistance was less than $400 \mathrm{ohms}$ per volt, terminal spacings were $1 \frac{11}{4}$ inch or more for 150 volts, and the coil stud spacings were 1 inch or more.

However, it is probable that at some relative humidity higher than 75 percent the surface resistivity of the panel of a volt box would become low enough to have a serious effect on the ratio.

\section{EFFECT OF SELF-HEATING}

For most of the volt boxes tested at the National Bureau of Standards during the past 13 years, it has been found that the ratio decreases during the first hour or two that rated voltage is applied, and remains nearly constant thereafter until the voltage is removed. The decrease is an exponential one, and the magnitude of the change for the 300 -volt range in volt boxes having a resistance of $200 \mathrm{ohms}$ per volt has been found to range from 0.005 percent to 0.03 percent, the average value being about 0.02 percent. One-half of this change occurs in about 20 minutes for most volt boxes.

6. H. Dike. U. S. Patent 2,026,616, issued Jan. 7, 1936. 
Two volt boxes having a resistance of $333 \mathrm{ohms}$ per volt and a maximum resistance per coil of $5,000 \mathrm{ohms}$ were found to show no measurable change in ratio even after several hours at rated voltage.

The 750-volt range of a volt box having $100 \mathrm{ohms}$ per volt showed a change in ratio of 0.04 percent in 2 hours at rated voltage.

Two factors are responsible for this self-heating effect. First, the resistance wire has an appreciable temperature coefficient of resistance; and second, for reasons of economy in construction the resistance coils in the higher voltage sections are usually on spools of approximately the same physical dimensions as those between the potentiometer taps, but have a much greater resistance per spool and hence a much greater temperature rise. The grade of manganin usually used in volt boxes has its maximum resistance near room temperature; and hence, as the higher resistance coils heat up, their resistance becomes a smaller percentage of the cold value than does that of the coils between the potentiometer taps. Hence, the voltage ratio of the volt box decreases.

Thermocouples were attached to the coils of a volt box of the usual construction having $200 \mathrm{ohms}$ per volt. It was found that for the 9,000- and 10,000-ohm coils the temperature rise was $10^{\circ} \mathrm{C}$ as compared with $4^{\circ} \mathrm{C}$ for the $300-\mathrm{ohm}$ coil. The ratio decreased with time on a smooth exponential curve for both the 150-volt range and the 300-volt range, the total change being 0.005 percent.

For volt boxes of normal construction the temperature rise of the coils is negligible at 20 percent of rated voltage, so that if the ratio of a volt box at rated voltage differs from that at 20 -percent voltage by an amount that is negligible for a given measurement then the heating effect will also be negligible for that measurement.

\section{APPENDIX I}

\section{Alternative Test Method}

A method which is simpler than that described in section II and which is for many purposes amply accurate for comparing the ratio $F_{X}$, of an unknown volt box with that, $\mathrm{F}_{S}$, of a standard volt box is shown in figure 9 . Here $H_{X}$ and $P_{X}$ are the resistances of the high-voltage side between $b$ and 4 and of the potentiometer side between $a$ and $b$, respectively, of the box under test, and $H_{S}$ and $P_{S}$ are the corresponding resistances of the standard. Hence, by definition (provided the resistances of the connections from 1 to $a$ and from $1^{\prime}$ to $d$ are negligible in comparison with $H_{X}+P_{X}$ and $H_{S}+P_{S}$, respectively),

$$
F_{X}=\frac{H_{X}+P_{X}}{P_{X}} \text { and } F_{s}=\frac{H_{s}+P_{s}}{P_{X}} .
$$

The galvanometer is first connected between the terminals 2 and $2^{\prime}$ and the slider between 1 and $1^{\prime}$ is adjusted to give a balance. This assures that the resistances of the two sides of the rheostat, together with the leads to $a$ and to $d$, are in the ratio

$$
\frac{L_{X}}{L_{s}}=\frac{P_{X}+H_{x}+R_{X}}{P_{s}+H_{s}+R_{s}}=\frac{i_{s}}{i_{X}} .
$$

The galvanometer is then shifted to join 3 and $3^{\prime}$, and one of the auxiliary resistors $R_{S}$ or $R_{X}$ as needed is increased until a balance is again reached. This gives the relation

$$
\frac{L_{x}+P_{x}}{L_{s}+P_{s}}=\frac{H_{x}+R_{x}}{H_{s}+R_{s}}=\frac{i_{s}{ }^{\prime}}{i_{x}{ }^{\prime}} .
$$


When by successive adjustment a balance is obtained for both positions of the galvanometer

$$
\frac{i_{X}}{i_{s}}=\frac{i_{x^{\prime}}}{i_{s}{ }^{\prime}},
$$

so that

$$
\frac{P_{x}}{P_{s}}=\frac{H_{x}+P_{x}+R_{x}}{H_{s}+P_{s}+R_{s}}
$$

Whence

$$
F_{x}=F_{s}\left[\frac{\left(1+\frac{R_{s}}{H_{s}+P_{s}}\right)}{\left(1+\frac{R_{X}}{H_{x}+P_{x}}\right)}\right] .
$$

In actual practice $R_{X}$ or $R_{S}$ is left zero according as $F_{X}$ is greater or less than $F_{S}$, so that the formula becomes very simple. This procedure does not fully eliminate the effect of the connections between points 1 and $a$ and between $1^{\prime}$ and $d$, but such

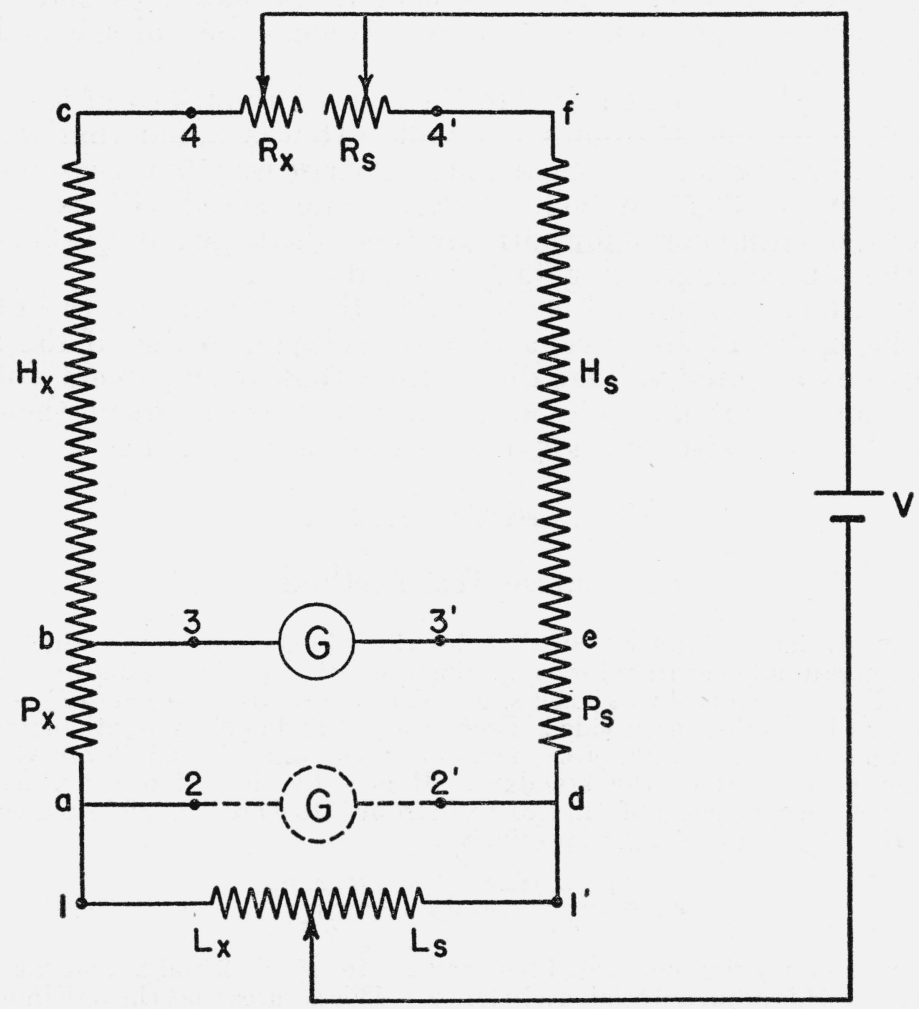

FigdRe 9.-Schematic diagram of circuit for intercomparison of volt boxes by the alternative method.

lead resistances are usually quite negligible. It also requires the measurement, though with only very moderate precision, of $H_{S}+P_{S}$ (or $H_{X}+P_{X}$ ). For boxes of different resistances a wide range of values of $R_{X}$ (or $R_{S}$ ) is required. 


\section{APPENDIX II}

\section{Derivation of Test Schedule for Calibrating the Standard Volt Box}

The working circuit of the standard volt box can be considered as made up of a succession of groups each made up of a number of sections. The first five sections of any one group have nominally equal resistances, and the last section has a resistance nominally five times this value. The first (counting from the low-potential end) section of each group is formed by all the preceding groups connected in series. Any section therefore can be identified by specifying two integers, the first, $m$, indicating the group of which the section is part, and the second, $k$, indicating the position of the section in its group.

If the Wheatstone bridge used in the successive measurements of the first five sections of the $m$ th group has the fixed ratio $B_{m} / A_{m}$, then the resistance of the $k$ th section is given by

$$
R_{m, k}=\frac{B_{m}}{A_{m}} p_{m, k},
$$

where $p_{m, k}$ is the resistance of the rheostat arm when measuring section $(m, k)$. Hence

$$
R_{m, k}=R_{m, 1}+\frac{B_{m}}{A_{m}}\left(p_{m, k}-p_{m, 1}\right)=R_{m, 1}+\frac{B_{m}}{A_{m}}\left(n_{m}, k\right),
$$

where $n_{m}, k$ is defined as $\mathrm{p}_{m},{ }_{k}-p_{m},{ }_{1}$. The total resistance of the first five sections of the group is given by

$$
\sum_{k=1}^{k=5}\left(R_{m, k}\right)=5 R_{m, 1}+\frac{B_{m}}{A_{m}} \sum_{k=1}^{k=5}\left(n_{m, k}\right) .
$$

The bridge ratio is now changed to $A^{\prime}{ }_{m} / B^{\prime}{ }_{m}$ and the bridge is balanced first across the combination of the first five sections in series giving

$$
\sum_{k=1}^{k=5}\left(R_{m, k}\right)=\frac{B_{m}{ }^{\prime}}{A_{m}{ }^{\prime}} p_{m, \text { 。 }}
$$

and then across the sixth section giving

Hence

$$
R_{m, t}=\frac{B_{m}^{\prime}}{A_{m}^{\prime}} p_{m, t}
$$

$$
R_{m, t}-\sum_{k=1}^{k=5}\left(R_{m, k}\right)=\frac{B_{m}{ }^{\prime}}{A_{m}{ }^{\prime}}\left(p_{m, t}-p_{m, s}\right) \text {. }
$$

We may write $p_{m, t}-p_{m, s}=n_{m, t}$ and combine eq 6 with eq 3 to get

$$
R_{m, \ell}=5 R_{m, 1}+\frac{B_{m}}{A_{m}} \sum_{k=1}^{k=5} n_{m, k}+\frac{B_{m}^{\prime}}{A_{m}^{\prime}} n_{m, t}
$$

The resistance of the entire $m$ th group is therefore

$$
\sum_{k=1}^{k=5} R_{m, k}+R_{m, t}=10 R_{m, 1}+2 \frac{B_{m}}{A_{m}} \sum_{k=1}^{k=5} n_{m}+\frac{B_{m}{ }^{\prime}}{A_{m}{ }^{\prime}} n_{m, t} .
$$

A very convenient choice of bridge ratios, which keeps $p$ nominally at 5,000 or 2,500 ohms, is to make

$$
\frac{B_{m}}{A_{m}}=10^{m-3} \text { and } \frac{B_{m}{ }^{\prime}}{A_{m}{ }^{\prime}}=10^{m-2}
$$

Also, as an abbreviation, we may write

$$
q_{m, 5}=\sum_{k=1}^{k=5} n_{m, k}
$$


Inserting these values and noting that this total resistance of the $m$ th group is identical with the resistance of the first section of the $(m+1)$ th group we get

$$
R_{(m+1,1)}=10 R_{m, 1}+2 \times 10^{m-3} q_{m, 5}+10^{m-2} n_{m, t}
$$

as the equation connecting resistance values in successive groups.

If this relation is applied successively, beginning with the first group $(m=1)$ we get for the resistance of the first section of the $m$ th group in terms of the resistance of the first section of the first group and the observed differences

$$
\begin{aligned}
R_{m, 1}=10^{m-1} R_{1,1} & +10^{m-3} n_{m-1, t}+2 \cdot 10^{m-4} q_{m-1,5} \\
& +10^{m-4+1} n_{m-2, t}+2 \cdot 10^{m-5+1} q_{m-2,5} \\
& +10^{m-5+2} n_{m-3, t}+2 \cdot 10^{m-6+2} q_{m-3,5}
\end{aligned}
$$

Simplifying this we get

$$
\begin{aligned}
& R_{m, 1}=10^{m-1} R_{1,1}+2 \cdot 10^{m-4}\left(q_{1,5}+q_{2,5} \ldots q_{m-1,5}\right) \\
& +10^{m-3}\left(n_{1, t}+n_{2, t}+\ldots \ldots n_{m-1, t}\right) .
\end{aligned}
$$

The total resistance $S_{m}, k$ up to the $k$ th tap in the $m$ th group is derived from eq 2

$$
S_{m, k}=\sum_{k^{\prime}=1}^{k^{\prime}=k} R_{m, k^{\prime}}=k R_{m, 1}+\frac{B_{m}}{A_{m}} \sum_{k^{\prime}=1}^{k^{\prime}=k} n_{m}, k^{\prime}
$$

Defining $q_{m},,_{k}$ as $\sum_{k^{\prime}=1}^{k^{\prime}=k} n_{m, k^{\prime}}$ and inserting the values of ratio from eq 9 , we get

$$
S_{m, k}=k R_{m, 1}+10^{m-3} q_{m, k}
$$

or by eq 11

$$
\begin{aligned}
S_{m, k} & =k \cdot 10^{m-1} R_{1,1}+2 \cdot 10^{m-4} k\left(q_{1,5}+q_{2,5} \cdots q_{m-1,5}\right) \\
& +10^{m-3} k\left(n_{1, t}+n_{2, t} \cdots n_{m-1, t}+\frac{q_{m}, k}{k}\right)
\end{aligned}
$$

If the circuit is used as a volt box with the first section as the low-voltage side and the total resistance to the $k$ th tap of the $m$ th group as the high side, the actual ratio is $S_{m, k} / R_{1,1}$ and the nominal value is $k \cdot 10^{m-1}$. The difference between these two values expressed in parts per million is, from eq 14

$$
\begin{gathered}
s_{m, k}=\frac{10^{8}}{k \cdot 10^{m-1}}\left(\frac{S_{m, k}}{R_{1,1}}-k \cdot 10^{m-1}\right)=\frac{2,000}{R_{1,1}}\left(q_{1,5}+q_{2,5} \cdots q_{m-1,5}\right) \\
+\frac{10,000}{R_{1,1}}\left(n_{1, t}+n_{2,5} \cdots \cdots n_{m-1, t}+\frac{q_{m}, k}{k}\right) .
\end{gathered}
$$

The nominal value of $R_{1,1}$ is $50 \mathrm{ohms}$, so that

$$
s_{m, k}=40\left(q_{1,5}+q_{2,5} \cdots q_{m-1,5}\right)+200\left(n_{1, t}+n_{2, t} \cdots n_{m-1, t}\right)+\frac{200}{k} q_{m, k} .
$$

When the standard volt box is calibrated, it is usually desirable to obtain the corrections for all the taps in a single process. For this purpose the schedule of computation indicated in table 1 has been derived by, in effect, applying eq 16 to consecutively increasing values of $k$ and of $m$. The quantities designated as $r_{m}, k$ constitute the last term in eq 16, and the summations indicated by the earlier terms are automatically made by the successive steps in computation. 
TABLE 1.-Schedule for computing corrections for the standard volt box

\begin{tabular}{|c|c|c|c|c|c|c|c|}
\hline $\begin{array}{l}\text { Section in } \\
\text { bridge arm }\end{array}$ & $\begin{array}{l}\text { Bridge } \\
\text { ratio a }\end{array}$ & $\begin{array}{l}\text { Ad- } \\
\text { just- } \\
\text { able } \\
\text { arm }\end{array}$ & $n$ 's & $q$ 's & $r$ 's & Correction & Range \\
\hline 1 & 2 & 3 & 4 & 5 & 6 & 7 & 8 \\
\hline $0-0.15$ & \multirow[b]{2}{*}{$100 / 10,000$} & $p_{1,1}$ & $\begin{array}{l}n_{1,1}=p_{1,1}-p_{1,1} \\
=0\end{array}$ & & & $s_{1,1}=0$ & $0-0.15$ \\
\hline $\begin{array}{l}0.15-0.30 \\
0.30-0.45 \\
0.45-0.60 \\
0.60-0.75\end{array}$ & & $\left\{\begin{array}{l}p_{1,2} \\
p_{1,3} \\
p_{1,4} \\
p_{1,3}\end{array}\right.$ & $\begin{array}{l}n_{1,2}=p_{1,2}-p_{1,1} \\
n_{1,3}=p_{1,3}-p_{1,1} \\
n_{1,4}=p_{1,4}-p_{1,1} \\
n_{1,5}=p_{1,5}-p_{1,1}\end{array}$ & $\begin{array}{l}q_{1,2}=n_{1,2} \\
q_{1,3}=q_{1,2}+n_{1,3} \\
q_{1,4}=q_{1,3}+n_{1,4} \\
q_{1,5}=q_{1,4}+n_{1,5}\end{array}$ & $\begin{array}{l}r_{1,2}=100 q_{1,2} \\
r_{1,3}=67 q_{1,3} \\
r_{1,4}=50 q_{1,4} \\
r_{1,5}=40 q_{1,5}\end{array}$ & $\begin{array}{l}s_{1,2}=r_{1,2} \\
s_{1,3}=r_{1,3} \\
s_{1,4}=r_{1,4} \\
s_{1,5}=r_{1,5}\end{array}$ & $\begin{array}{l}0-0.30 \\
0-0.45 \\
0-0.60 \\
0-0.75\end{array}$ \\
\hline \multirow{2}{*}{ 0. $\begin{array}{r}0-0.75-1.5 \\
\text { (1) }\end{array}$} & \multirow{6}{*}{$1,000 / 10,000$} & $p_{1, \varepsilon}$ & & & & & \\
\hline & & $p_{1, t}$ & $n_{1, t}=p_{1, t}-p_{1, s}$ & $q_{1, t}=n_{1, t}$ & $r_{1, t}=200 q_{1}, t$ & $s_{2,1}=s_{1,5}+r_{1}, t$ & $0-1.5$ \\
\hline $\begin{array}{r}0-1.5 \\
1.5-3.0\end{array}$ & & $\left\{\begin{array}{l}p_{2,1} \\
p_{2,2}\end{array}\right.$ & $n_{2,2}=p_{2}$ & $g_{2,2}=n$ & $r_{2,2}=100 q_{2,2}$ & $s_{2}=s_{2}+r_{2}$ & $0-30$ \\
\hline $3.0-4.5$ & & $\mid \begin{array}{l}p_{2,2} \\
p_{2,3}\end{array}$ & $n_{2,3}=p_{2,3}-p_{2,1}$ & $\begin{array}{l}q_{2,3}=q_{2,2} \\
q_{2,2}+n_{2,3}\end{array}$ & $\begin{array}{l}T_{2,2}=100 q_{2,2} \\
r_{2,3}=67 q_{2,3}\end{array}$ & $\begin{array}{l}s_{2,2}=s_{2,1}+T_{2,2} \\
s_{2,3}=s_{2,1}+r_{2,3}\end{array}$ & $0-4.5$ \\
\hline 4. $5-6.0$ & & $p_{2,4}$ & $n_{2,4}=p_{2,4}-p_{2,1}$ & $q_{2,4}=q_{2,3}+n_{2,4}$ & $r_{2,4}=50 q_{2,4}$ & $s_{2,4}=s_{2,1}+r_{2,4}$ & $0-6.0$ \\
\hline $6.0-7.5$ & & $p_{2,5}$ & $n_{2,5}=p_{2,5}-p_{2,1}$ & $q_{2,5}=q_{2,4}+n_{2,5}$ & $r_{2,5}=40 q_{2,5}$ & $s_{2,5}=s_{2,1}+r_{2,5}$ & $0-7.5$ \\
\hline \multirow{6}{*}{$\begin{array}{c}0-7.5 \\
7.5-15 \\
0-15 \\
15-30 \\
30-45 \\
45-60 \\
60-75\end{array}$} & \multirow{6}{*}{$1,000 / 1,000$} & $p_{2, \varepsilon}$ & & & & & \\
\hline & & $\begin{array}{l}p_{2, t} \\
p_{3,1}\end{array}$ & $n_{2, t}=p_{2, t}-p_{2, s}$ & $q_{2, t}=n_{2, t}$ & $r_{2, t}=200 q_{2, t}$ & $s_{3,1}=s_{2,5}+r_{2, t}$ & $0-15$ \\
\hline & & $\left\{p_{3,2}\right.$ & $n_{3,2}=p_{3,2}-p_{3,1}$ & $q_{3,2}=n_{3}$ & $r_{3.2}=100 q_{3,2}$ & $s_{3,2}=s_{3,1}+r_{3,2}$ & $0-30$ \\
\hline & & $p_{3,3}$ & $n_{3,3}=p_{3,3}-p_{3,1}$ & $q_{3,3}=q_{3,2}+n_{3,3}$ & $r_{3.3}=67 q_{3.3}$ & $s_{3,3}=s_{3,1}+r_{3}, 3$ & $0-45$ \\
\hline & & $p_{3.4}$ & $n_{3,4}=p_{3,4}-p_{3,1}$ & $q_{3,4}=q_{3,3}+n_{3,4}$ & $r_{3.4}=50 q_{3.4}$ & $s_{3,4}=s_{3,1}+r_{3,4}$ & $0-60$ \\
\hline & & $\left(p_{3,5}\right.$ & $n_{3.5}=p_{3.5}-p_{3.1}$ & $q_{3.5}=q_{3.4}+n_{3.5}$ & $r_{3.5}=40 q_{3.5}$ & $s_{3,5}=s_{3,1}+r_{3,5}$ & $0-75$ \\
\hline \multirow{2}{*}{$\begin{array}{c}0-75 \\
75-150\end{array}$} & \multirow{6}{*}{$10,000 / 1,000$} & $p_{3,8}$ & & & & & \\
\hline & & $p_{3, t}$ & $n_{3, t}=p_{3, t}-p_{3, s}$ & $q_{3, t}=n_{3, t}$ & $r_{3, t}=200 q_{3, t}$ & $s_{4,1}=s_{3,5}+r_{3, t}$ & $0-150$ \\
\hline $\begin{array}{r}0-150 \\
150-300\end{array}$ & & $p_{4,1}$ & & & & & \\
\hline $\begin{array}{l}150-300 \\
300-450\end{array}$ & & $\left|\begin{array}{l}p_{4,2} \\
p_{4,3}\end{array}\right|$ & $\begin{array}{l}n_{4,2}=p_{4,2}-p_{4,1} \\
n_{4,3}=p_{4,3}-p_{4,1}\end{array}$ & $\begin{array}{l}q_{4,2}=n_{4,2} \\
q_{4,3}=q_{4,2}+n_{4,3}\end{array}$ & $\begin{array}{l}r_{4,2}=100 q_{4,2} \\
r_{4,3}=67 q_{4,3}\end{array}$ & $\begin{aligned} s_{4,2}=\boldsymbol{s}_{4,1}+r_{4,2} \\
\boldsymbol{s}_{4,3}=\boldsymbol{s}_{4,1}+r_{4,3}\end{aligned}$ & $\begin{array}{l}0-300 \\
0-450\end{array}$ \\
\hline $450-600$ & & $p_{4,4}$ & $n_{4,4}=p_{4,4}-p_{4,1}$ & $q_{4,4}=q_{4,3}+n_{4,4}$ & $r_{4,4}=50 q_{4,4}$ & $s_{4,4}=s_{4,1}+r_{4,4}$ & $0-600$ \\
\hline $600-750$ & & $p_{4,3}$ & $n_{4,5}=p_{4,5}-p_{4,1}$ & $q_{4,5}=q_{4,4}+n_{4,5}$ & $r_{4,5}=40 q_{4,5}$ & $s_{4,5}=s_{4,1}+r_{4,5}$ & $0-750$ \\
\hline
\end{tabular}

- In figure 4 ( $\mathrm{A}$ and $\mathrm{B}$ ) the bridge ratio is $B / A$.

Washington, June 6, 1941. 